\section{Neuer Hoffnungsschimmer für die renale Denervation}

\begin{abstract}
Nach anfänglichem Hype war es um die renale Denervation zwischenzeitlich sehr still geworden. Doch nun kehrt die interventionelle Methode zur Blutdrucksenkung mit einer erfolgreich verlaufenden Studie zurück auf die große Kongressbühne.
\end{abstract}

$\mathrm{N}$ achdem viele die renale Denervation als vermeintlich gescheiterten neuen Therapieansatz schon zu Grabe getragen hatten, keimt nun wieder Hoffnung auf ihre Wiederbelebung. Eine randomisierte kontrollierte Studie kommt zu dem Ergebnis, dass sich ein erhöhter Blutdruck mit dieser interventionellen Methode signifikant stärker senken ließ als mit einer Scheinprozedur („sham“) - und zwar bei Patienten mit relativ moderater Hypertonie ohne jegliche antihypertensive Medikation.

\section{"Proof of Principle"}

Prof. Michael Böhm aus Homburg/Saar hat Zwischenergebnisse von 80 Teilnehmern dieser SPYRAL HTN-OFF MEDStudie beim ESC-Kongress vorgestellt. Der Kardiologe interpretiert die Ergebnisse als „Proof of Principle“ für die Effektivität der renalen Denervation.

Diese Effektivität der Denervation sympathischer Nerven in der Nierenarterienwand mithilfe eines Multielektroden-Katheters kam dabei sowohl bei der ambulanten 24-Stunden-Messung als auch bei der Praxismessung zum Ausdruck. Bei der ambulanten Messung wurde im Vergleich zur Kontrollgruppe nach drei Monaten eine Abnahme des systolischen und diastolischen Blutdrucks um 5 und 4,4 mmHg in Relation zum Ausgangswert festgestellt. Bei der Praxismessung betrugen die entsprechenden Blutdruckabnahmen 7,7 und 4,9 mmHg. Alle Blutdruckveränderungen erwiesen sich als signifikant. In puncto Sicherheit waren keine Probleme zu vermelden.

Diese Ergebnisse sind ein neuer Hoffnungsschimmer in der wechselvollen Geschichte der renalen Denervation. Beeindruckende Effekte auf den Blutdruck, die anfänglich in zumeist unkontrollierten Studien beobachtet wurden, hatten Begeisterung für die Methode ausgelöst.
Umso größer war die Enttäuschung, als die Ergebnisse von SYMPLICITY HTN-3 als bis dato methodisch beste Studie mit einer Scheinprozedur als Kontrolle bekannt wurden. Die renale Denervation hatte im Vergleich dazu keine signifikant besseren Therapieergebnisse zu bieten.

\section{SYMPLICITY HTN-3 - das letzte Wort ist nicht gesprochen}

Für einige Experten gab es bei dieser Studie aber zu viele Fragzeichen. So erinnerte Böhm daran, dass es hinsichtlich der Medikation Veränderungen bei der Therapieadhärenz gegeben habe. Die Denervation der sympathischen Nerven in der Wand der Nierenarterien sei häufig inkomplett gewesen.

Und auch die Unerfahrenheit vieler Studienärzte mit der Methode sowie die Selektion von Patienten mit isolierter systolischer Hypertonie, die nicht gut auf die Denervation ansprechen, wurden als Limitierungen angesehen.
Lehren für den Neubeginn

Daraus hat man Lehren für einen Neubeginn gezogen. Diese prägen das Konzept der SPYRAL HTN-OFF MED-Studie. Erstmals sind Patienten mit relativ moderater Hypertonie rekrutiert worden, die bislang keine Antihypertensiva eingenommen hatten oder diese für die Dauer der Studie absetzten. Ihr systolischer Blutdruck musste im Bereich zwischen 150 und $180 \mathrm{mmHg}$ (Praxismessung) oder zwischen 140 und $170 \mathrm{mmHg}$ (ambulante 24-Stunden-Messung) liegen - bei diastolischen Werten von 90 mmHg oder höher.

Anhand von Blut- und Urinmessungen wurde kontrolliert, ob die Nichteinnahme der Medikamente tatsächlich eingehalten wurde. Alle Teilnehmer wurden einer renalen Angiografie unterzogen, dann erfolgte die Zuteilung zu den beiden Behandlungsgruppen. Die Angiografie bildete die Scheinprozedur. Zur möglichst vollständigen Denervation - sie erfolgte auch in Seitenästen der Nierenarterien - wurde ein spiralförmiges Multielektroden-System verwendet.

Peter Overbeck

Quelle: Hot Line: Late-Breaking Clinical Trials 2 "SPYRAL HTN OFF-MED Study", ESC-Kongress, 28.8.2017 in Barcelona.

Townsend RR et al. Catheter-based renal denervation in patients with uncontrolled hypertension in the absence of antihypertensive medications (SPYRAL HTN-OFF MED): a randomised, sham-controlled, proof-of-concept trial. Lancet, online 28. August 2017, https://doi.org/10.1016/ S0140-6736(17)32281-X

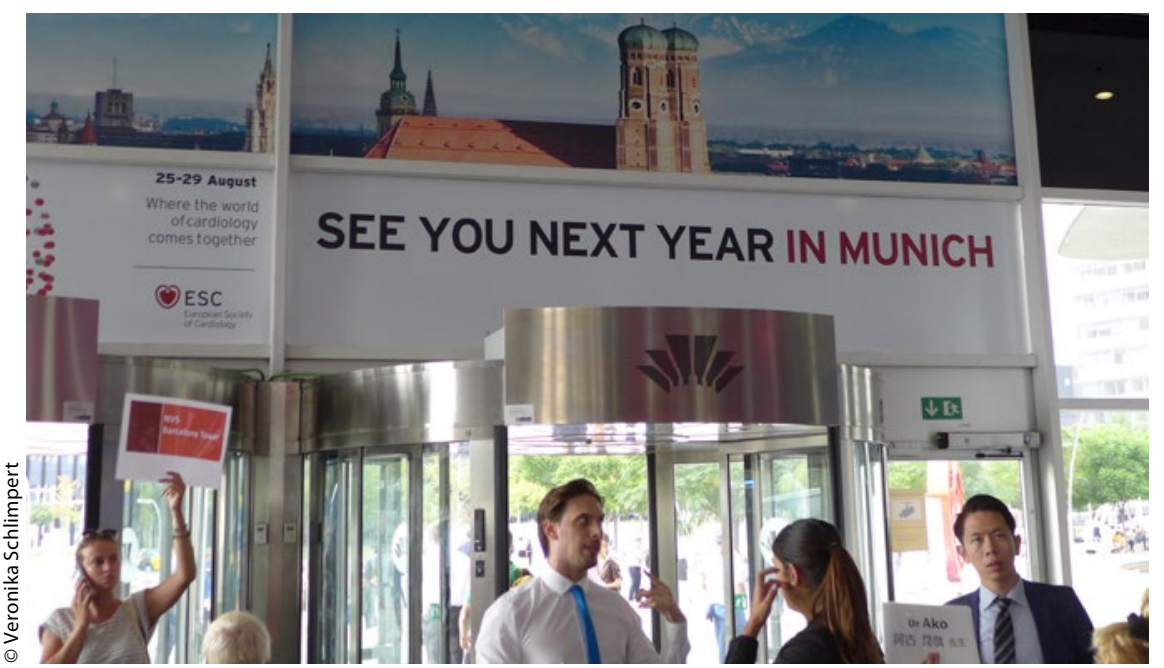

Nächstes Jahr dann in München; der Europäische Kardiologen-Kongress zählt mit über 30.000 Besuchern zu den größten Medizin-Kongressen der Welt. 